\title{
An Existential Study on Structural, Optical and Electronic Properties of ZnO Nanoparticles and Nanorods
}

\author{
* Anju Singh ${ }^{1}$, Dr. H.L. Vishwakarma ${ }^{2}$ \\ ${ }^{1,2}$ Dept of Applied Physics, Rungta College of Engg. \& Tech., Kohka, Kurud Road Bhilai(C.G)
}

\begin{abstract}
Research interest in $\mathrm{ZnO}$ nanostructure derives from their excellent luminescent properties and availability of low cost fabricating and processing, which hold promise for the development of electronic and optoelectronic devices. In this work, the synthesis routes for zinc oxide $(\mathrm{ZnO})$ nanoparticles and nanorods, their structural, optical \& electronic properties have been established. ZnO has many potential applications like sunscreens,biosensors,food additives,pigments, rubber manufacture optoelectronic, piezoelectric, spintronic devices, field effect transistors(FET)etc.ZnO nanoparticles and nanorods have been received more attention in new age material science. In this paper we discuss $\mathrm{ZnO}$ is useful for other semiconductor materials and their properties are different from others.
\end{abstract}

Key words: Nanorods, optical and Electronic properties, spintronics, nanostructure

\section{Introduction}

The world of semiconductor materials has enormous affect on the economy. In this case, there are two popular examples of semiconductor materials ( $\mathrm{Ge}$ and $\mathrm{Si}$ ) which are available for different purposes. Germanium gets popular due to possession of property such as low melting point and lack of natural occurring germanium oxide to avoid the surface from electrical leakage where as silicon dominates the commercial market for its better fabrication technology and application to integrate circuits for different reasons. But the rapid growing world needs speed along with technology. This requirement was well fulfilled by GaAs, which easy the path for the design of high speed and optoelectronic devices.GaAs is a direct band gap semiconductor which possessing higher carrier mobility and higher effective carrier velocity in comparison to Si makes it better suited for optoelectronic devices.

The world now demands a material that should possess fundamental properties such as larger band gap, higher electron mobility as well as higher breakdown field strength. Zinc Oxide fulfils the required properties. Zinc Oxide materials have remarkable attention due to its wide range of application in ultraviolet(UV) lasers, power generators, solar cells, gas sensors, field emission devices, capacitors, varistors, transparent UV resistance coating,photoprinting,electrophotography,electrochemical nanodevices, sunscreen lotion, cosmetic and medicated creams etc.

$\mathrm{ZnO}$ is II-VI group wide band gap $(3.37 \mathrm{eV})$ semiconductor having a large electron hole binding energy of $60 \mathrm{meV}$ would allow for excitonic transitions even at room temperature, which could mean high radiative recombination efficiency for spontaneous emission as well as a lower threshold voltage for laser emission. The most important applications of zinc oxide in different field such as electronics, optics, optoelectronics, laser and light emitting diode. The piezoelectric and pyroelectric properties of $\mathrm{ZnO}$ make it a great candidate for sensors, transducers, energy generators and photo catalysis for hydrogen production. $\mathrm{ZnO}$ is also a green material that is useful for bio compatible, biodegradable and bio safe for medical applications and environmental science.

\section{Methods for Synthesis of Nanomaterials}

Properties of nanomaterials are dependent on their shape, size and uniformity. Hence synthesis of nanostructures of uniform shape and size is very essential for basic studies as well as for their potential applications. Synthesis of nanostructure of uniform shape and size is one of the most challenging missions in the area of nanotechnology.

Normally there are two approaches to synthesize nanomaterial such as "top down" and "bottom up" approach. In a top down approach, a bulk material is taken and by using suitable methods, commonly physical methods, size is decreased to required nanometer range. In bottom up approach, however, the synthesis process starts in the atomic or molecular level. Atoms or molecules of required materials are allowed to gather till size of the material gets increased to the required nanometer range. Bottom up approach mostly relies on chemical process. Some of the famous methods that come in the category of top down approach are high energy milling,electro-explosion,laser ablation, sputtering, molecular beam epitaxy, vapour condensation and low energy cluster beam deposition.

Synthesis of nanomaterial is based on three schemes i.e. 
(1)Liquid phase synthesis

(2)Gas phase synthesis

(3)Vapour phase synthesis

(1)Liquid phase synthesis:-This synthesis applies chemical reactions in solvent. This leads to colloids, in which the nanoparticles formed can be stabilized against aggregation by surfactants.

I. Co-Precipitation

II. Sol Gel processing

III. Micro emulsion synthesis

IV. Hydrothermal/Solvothermal synthesis

V. Microwave synthesis

VI. Sonochemical synthesis

VII. Template synthesis

(2)Gas phase synthesis:-Super saturation achieved by vaporizing material into a background gas, then cooling the gas.

(3) Vapour phase synthesis:-
A. Inert gas condensation
B. Pulsed laser ablation
C. Spark discharge generation
D. Ion sputtering
E. Chemical vapour synthesis
F. Spray pyrolysis
G. Laser pyrolysis/photochemical synthesis
H. Thermal plasma synthesis
I. Flame synthesis
J. Flame spray pyrolysis
K. Low temperature reactive synthesis

Nanostructure materials can have remarkably different properties depending on the chosen synthesis technique. Each method provides some benefits over other methods while suffering limitation from others.

\section{Basic Physical Parameters and Structural Properties}

$\mathrm{ZnO}$ is an II-VI compound semiconductor whose iconicity lies at the borderline between the covalent and ionic semiconductors. The crystal structures shared by $\mathrm{ZnO}$ are wurtzite (B4), zinc blende (B3) and rock salt (or Rochelle salt)(B1) as shown in fig.1.Under atmosphere conditions, the thermodynamically stable phase is that of wurtzite symmetry. The zinc blende $\mathrm{ZnO}$ structure can be stabilized only by growth on cubic substrates [1-3] and the rock salt or Rochelle salt $(\mathrm{NaCl})$ structure may be obtained at relatively high pressure [4].

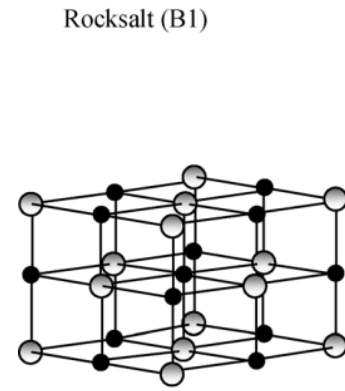

(a)

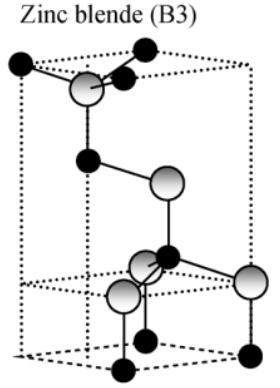

(b)
Wurtzite (B4)

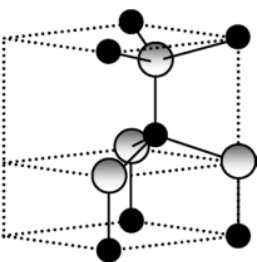

Fig.1 Stick-and-ball representation of ZnOcrystal structures:

(a) Cubic rocksalt (B1), (b) cubic zinc blende (B3), and

(c) Hexagonal wurtzite (B4). Shaded gray and black spheres denote Zn and O atoms, respectively.

In an ideal wurtzite crystal structure has a hexagonal unit cell with two lattice parameters a and $\mathrm{c}$ where $\mathrm{a}=3.2495 \mathrm{~A}^{0}$ and $\mathrm{c}=5.2096 \mathrm{~A}^{0}$ in the ratio of $\mathrm{c} / \mathrm{a}=1.602$ and the density is $5.605 \mathrm{gcm}^{-3}$.It belongs to the space group $C^{4}{ }_{6 V}$ in the Schoenflies notation and P63mc in the Hermann-Mauguin notation and distinguished by two interconnecting sub lattices of $\mathrm{Zn}^{2+}$ and $\mathrm{O}^{2-}$ where each anion is enclosed by four cations at the corners of a tetrahedron through $\mathrm{SP}^{3}$ covalent bonding. This tetrahedral coordination gives rise to polar symmetry along the hexagonal axis. This polarity is responsible for a number of properties of $\mathrm{ZnO}$, including its piezoelectricity and 
spontaneous polarization and is also a key factor in crystal growth, etching and defect generation. The four most common face terminations of wurtzite $\mathrm{ZnO}$ are the polar $\mathrm{Zn}$ terminated (0001) and $\mathrm{O}$ terminated (0001)

faces(c-axis oriented) and the non polar (1120)(a-axis) and (1010) faces which both contain an equal number of $\mathrm{Zn}$ and $\mathrm{O}$. The polar faces are known to posses different chemical and physical properties and the Oterminated face possess a slightly different electronic structure to the three faces [5]. The schematic diagram of wurtzite $\mathrm{ZnO}$ structure is shown in fig.2. The structure is composed of two interpenetrating hexagonal closed packed (hcp) sublattices, each of which include of one type of atom displaced with respect to each other along the three fold $\mathrm{c}$-axis by the amount of $\mathrm{u}=3 / 8=0.375$ (in an ideal wurtzite structure) in fractional coordinates.

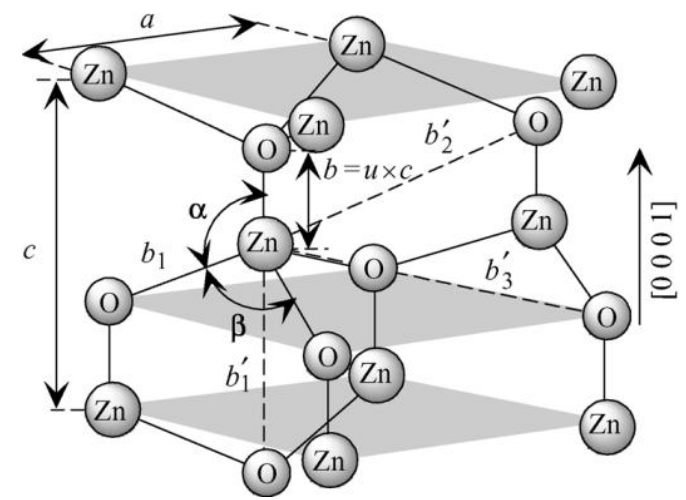

Fig.2: Schematic representation of a wurtzitic $\mathrm{ZnO}$ structure with lattice constants a in the basal plane and $\mathrm{c}$ in the basal direction, $\mathrm{u}$ parameter, which is expressed as the bond length or the nearest-neighbor distance $\mathrm{b}$ divided by c.

In a real $\mathrm{ZnO}$ crystal the wurtzite structure deviates from the ideal arrangement, by changing the c/a ratio or the $u$ value. This deviation occurs such that the tetrahedral distances are kept roughly constant in the lattice. Experimentally, for wurtzite $\mathrm{ZnO}$, the real values of $\mathrm{u}$ and c/a were determined in the range $\mathrm{u}=0.3817$ to 0.3856 and $\mathrm{c} / \mathrm{a}=1.593$ to $1.6035[6-8]$.

\section{Optical and Electronic Properties}

The more significant factor responsible for a material to show a better optoelectronic property is the large exciton binding energy and this property is possessed by zinc oxide having binding energy of $60 \mathrm{meV}$ which could be attended at and above room temperature due to excitonic recombination $[9,10,11,12-14]$.The process of optical absorption and emission have been affected by bound excitons which are extrinsic transitions related to dopants or defects thereby usually responsible for creating discrete electronic states in the band gap.The excitons of the wurtzite $\mathrm{ZnO}$ exist in various forms, which can be divided into free and bound excitons. The free excitons(FX) which dominate in high quality samples with low impurity concentrations are free to move without any constraints. FX can also exhibit excited states, in addition to their ground state transitions. Whereas, in the samples with high impurity concentration,the dopants or defects usually create discrete electronic states in the band gap and thus affect the optical performance of samples.In this case the excitons could be bounded to kinds of impurities, that is , neutral or changed donors and acceptors.

\section{Energy Band Gap}

The electronic band structure of $\mathrm{ZnO}$ has been calculated by a number of groups [15-21].The results of a band structure calculation using the Local Density Approximation (LDA) and incorporating atomic selfinteraction corrected pseudo potential(SIC-PP) to accurately account for the $\mathrm{Zn} 3 \mathrm{~d}$ electrons[21].As reported from various literatures the band gap of $\mathrm{ZnO}$ films mostly depend on the carrier concentration and is found to be $3.37 \mathrm{eV}$ on basis of carrier concentration of 1018-1020/c. $\mathrm{m}^{3}$.Increase in band gap when concentration changes to 3-4 x 1019/c.m ${ }^{3}$.Quantum confinement of electrons small grains created by potential barriers at the grain boundaries are thought to responsible for the drastic change in band gap. The $\mathrm{ZnO}$ having direct band gap is very well indicated by the valance band maxima and lowest conduction band minima both occurring at the same $\Gamma$ point of $\mathrm{k}=0 . \mathrm{Zn} 3 \mathrm{~d}$ levels are indicated by bottom ten bands and $\mathrm{O} 2 \mathrm{p}$ bonding states are highlighted by next six bands from $-5 \mathrm{eV}$ to $0 \mathrm{eV}$. The empty $\mathrm{Zn} 3 \mathrm{~s}$ levels signified by first two conduction band states are mainly $\mathrm{Zn}$ localized. Crystallization of $\mathrm{ZnO}$ mostly favourable in wurtzite symmetry and crystal field splitting as well as spin orbit interaction results in three states say A, B\&C in fig. 3 . 


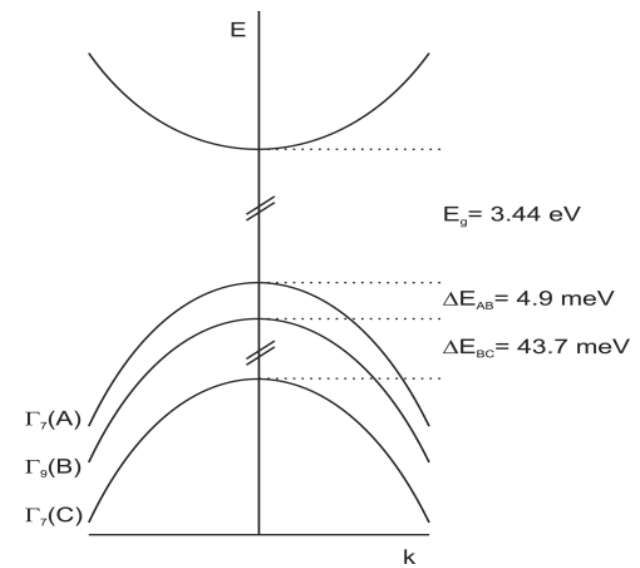

Fig.3:Schematic diagram representing the crystal-field and spin-orbit splitting of the valance band of $\mathrm{ZnO}$ into 3 sub band states $\mathrm{A}, \mathrm{B}$ and $\mathrm{C}$ at $4.2 \mathrm{~K}$.

\section{Applications of $\mathrm{ZnO}$ Nanorostructures}

Zinc Oxide due to its versatility and multifunctionality creates attention in the research field related to its applications. A wide number of synthesis techniques also been developed by which $\mathrm{ZnO}$ can be grown in different nanoscale forms and thereby different novel nanostructures can be fabricated with different shapes ranging from nanowires to nanobelts and even nanosprings. $\mathrm{ZnO}$ is widely used in our society, and indeed it is a key element in many industrial manufacturing process including paints,cosmectics,pharmaceutical,plastic, batteries, electrical equipments,rubber,soap,textiles. With improvements in growth technology of $\mathrm{ZnO}$ nanostructures, epitaxial layers, single crystals and nanoparticles. $\mathrm{ZnO}$ based nanostructures including nanowires arrays holds a host of opportunities for flat screen displays ,field emission sources,gas,chemical[22] and biological sensors and as UV light emitters and switches[22-25].Epitaxial layers and single crystals will be important for the development of optoelectronic(blue and ultraviolet light emitters and detectors)[26],piezoelectric[27] and spintronic[28] devices and together with GaN may form the light source of the $21^{\text {st }}$ century[29].

Normally, intrinsic $\mathrm{ZnO}$ is a n-type semiconductor, partly due to natural doping by interstitial hydrogen atom or oxygen vacancy [30].Many groups have reported that several kinds of metals with low work function, such as titanium [31-33], tungsten [34, 35] and niobium [36] and so forth, were used as electrons to form ideal ohmic contact with $\mathrm{ZnO}$, thereby $1 \mathrm{D} \mathrm{ZnO}$ nanostructures were applied as a conducting channel to construct field effect transistors (FET).

\section{Conclusion}

The structural, optical and electronic properties of $\mathrm{ZnO}$ nanoparticles and nanorods were examined. Nanostructural materials can have significantly different properties, depending on the chosen fabrication route. At present, there are many methods to examine various properties of the prepared sample, in which a number of characterization methods are used. Their results give the information about the different structural, optical and electronic properties of the sample. On the basis of their characterization we can use the material in the field of different applications.

\section{References}

[1] T. Kogure, Y. Bando, J. Electron Microsc. 47,1993,7903.

[2] A. B. M. A. Ashrafi, A. Ueta, A. Avramescu, H. Kumano, I. Suemune, Y. W. Ok, T. Y. Seong, Appl. Phys. Lett. 76,2000,550.

[3] S. K. Kim, S. Y. Seong, C. R. Cho, Appl. Phys. Lett. 82,2003,562.

[4] C. H. Bates,W. B. White, R. Roy, Science 137,1962,993.

[5] O. Dulub, L. A. Boatner, U. Diebold, Surf. Sci. 519,2002, 201.12

[6] E. H. Kisi, M. M. Elcombe, Acta Cryst. C45,1989,1867.

[7] J. E. Jaffe, A. C. Hess, Phys. Rev. B 48,1993,7903.

[8] L. Gerward, J. S. Olsen, J. Synchrotron Radiat. 2,1995, 233.

[9] Djurisic A,Leung Y.Optical properties of ZnO nanostructures.Small,2006,2:944-961

[10] Klingshirn C F.Semiconductor optics.2007

[11] Klingshirn C.ZnO:From basics towards applications.Physica Status Solidi(b),2007,244:4027-2073

[12] Klingshirn C,Priller H,Decker M,et al.Excitonic properties of ZnO.Adv Solid State Physics,2006,45:275-287

[13] Teke A,Ozgur U,Dogan S,et al.Excitonic fine structure and recombination dynamics in single-crystalline ZnO.Phys Rev B,2004,70:195-207

[14] Meyer B,Alves H,Hofmann D,et al.Bound exciton and donor-acceptor pair recombination in ZnO.Physica Status Solidi(b), 2004,241:231-260

[15] J. E. Jaffe, J. A. Snyder, Z. Lin, A. C. Hess, Phys. Rev. B 62,2000,1660.

[16] J. R. Chelikowsky, Solid State Commun. 22,1977, 351. 
[17] U. Rossler, Phys. Rev. 184,1969,733.

[18] S. Bloom, I. Ortenburger, Phys. Stat. Sol. (b) 58,1973,561.

[19] M. Usuda, N. Hamada, T. Kotani, M. van Schilfgaarde, Phys. Rev. B 66,2002,125101.

[20] I. Ivanov, J. Pollmann, Phys. Rev. B 24,1981, 7275.

[21] D. Vogel, P. Krüger, J. Pollmann, Phys. Rev. B 52,1995, R14316.

[22] L. Liao, J. C. Li, D. F. Wang, C. Liu, C. S. Liu, Q. Fu, L. X. Fan, Nanotech. 16, 2005,985.

[23] Y. Xia, P. Yang, Y. Sun, Y. Wu, B. Mayers, B. Gates, Y. Yin, F. Kim, H. Yan, Adv. Mater. 15,2003, 353.

[24] Z. L.Wang, J. Phys. Cond. Matter 16,2004, R829.

[25] D. J. Sirbuly, M. Law, H. Q. Yan, P. D. Yang, J. Phys. Chem. B 109 ,2005, 15190

[26] D. C. Look, Mater. Sci. Eng. (b) 80,2001, 383.

[27] J. G. E. Gardeniers, Z. M. Rittersma, G. J. Burger, J. Appl. Phys. 83,1998, 7844

[28] D. P. Norton, S. J. Pearton, A. F. Hebard, N. Theodoropoulou, L. A. Boatner, R. G.Wilson, Appl. Phys. Lett. 82,2003, 239.

[29] J. Nause, Comp. Semicond. 11,2005, 29.

[30] Ozgur U,Alivov Y I,Liu C,et al.A comprehensive review of ZnO materials and devices.J Appl Phys,2005,98:041301.

[31] Ip K,Heo Y,Baik K,et al.Carrier concentration dependence of Ti/Al/Pt/Au contact resistance on n-type ZnO,Appl Phys Lett,2004,84:544-546.

[32] Chang C Y,Tsao F C,Pan C J,et al. Electroluminescence from ZnO nanowire/polymer composite pn junction.Appl Phys Lett,2006,88:17350.

[33] Park W I,Kim J S,Yi G C,et al.Fabrication and electrical characteristic of high-performance ZnO nanorod field-effect transistors,Appl Phys Lett,2004,85:5052-5054

[34] Liu Y,Wang S,Zhang Z,et al.Measuring the electrical characteristics of individual junction in the SnO capped ZnO nanowire arrays on Zn substrate. Appl Phys Lett, 2008, 92:033102

[35] She J,Xiao Z,Yang Y,et al.Correlation between resistance and field emission performance of individual ZnO one-dimensional nanostructures.Acs Nano,2008,2:2015-2022

[36] Cha S,Jang J,Choi Y,et al.High performance $\mathrm{ZnO}$ nanowire field effect transistor using self aligned nanogap gate etrodes.Appl Phys Lett,2006,89:263102 